\title{
LA GESTIÓN DOCUMENTAL EN FUNCIÓN DE LA GOBERNANZA DE LA INFORMACIÓN. EL CASO DE LA UNIVERSIDAD CENTRAL "MARTA ABREU" DE LAS VILLAS
}

\author{
Asleni Díaz Jiménez ${ }^{1}$ \\ https://orcid.org/0000-0002-8073-0040 \\ Arlene González Pérez ${ }^{2}$ \\ https://orcid.org/0000-0003-2290-9648
}

\section{RESUMEN}

Recebido: 06.11 .2019

Aceito: 12.06 .2020

Publicado: 06.07.2020

La Gestión Documental enfocada desde la perspectiva de la Gobernanza de la Información, le proporciona a cualquier entidad universitaria una óptima gestión de todos sus procesos. Las tendencias internacionales apuntan a una integración entre los fundamentos teórico-prácticos existentes sobre Gestión Documental y la Gobernanza de Información. Mancomunar los modelos y principios básicos asociados a los procesos de evaluación documental es fundamental para logar una adecuada gestión organizacional. Son variados los autores que tratan esta temática, ya que los diagnósticos son aplicados por las diferentes instituciones cuando lo creen conveniente, destacando García (2012), Puerta (2016) y Mena \& García (2019). La Metodología empleada para la realización del diagnóstico es el Modelo de Gestión de Documentos y Administración de Archivos de la Red de Transparencia y Acceso a la Información publicado en enero del 2015. La realización del dictamen mediante dicha metodología comprueba que el estado de la Gestión Documental desde la óptica de la Gobernanza de la Información en la Universidad Central "Marta Abreu" de Las Villas se encuentra en un nivel muy elemental.

Palabras clave: Gestión Documental, Gobernanza de la Información, Modelo de Gestión de Documentos, Universidad Central "Marta Abreu" de Las Villas.

A gestão documental com base na governança da informação. O caso da Universidad Central "Marta Abreu" de las Villas.

\section{RESUMO}

A Gestão Documental focada desde a perspetiva da Governança da Informação, proporciona a qualquer entidade universitária uma óptima gestão de todos seus processos. As tendências internacionais apontam para uma integração entre os fundamentos teórico-prácticos existentes sobre a Gestão Documental e a Governança da Informação. O agrupamento de modelos e princípios básicos associados aos processos de avaliação documental é fundamental para conseguir uma adequada gestão organizacional. São vários os autores que tratam desta temática, pois os diagnósticos são aplicados pelas diferentes instituições quando consideram conveniente, destacando García (2012), Puerta (2016) e Mena\&García (2019). A Metodologia utilizada para a realização do diagnóstico é o Modelo de Gestão de Documentos e Administração de Arquivos da Rede de Transparência e Acesso à Informação publicado em Janeiro de 2015. A realização do relatório mediante dita metodologia comprova que o estado da Gestão Documental a partir da óptica da Governança da Informação na Universidad Central "Marta Abreu" de Las Villas se encontra num nível muito elemental.

Palavras chave: Gestão Documental, Governança da Informação, Modelo de Gestão de Documentos, Universidad Central "Marta Abreu" de Las Villas.

Documental management based on information governance. The case of the Universidad Central "Marta Abreu" in las Villas.

\section{ABSTRACT}

Document Management focused on the perspective of Information Governance, provides any university entity with optimal management of all its processes. International trends point to a uniqueness between the existing theoretical and practical foundations on Document Management and Information Governance. Combining the basic models and principles associated with the documentary evaluation processes is essential to achieve adequate organizational management. The authors that deal with this topic are varied, since the diagnoses are applied by the different institutions when they consider it convenient, highlighting García (2012), Puerta (2016) and Mena \& García (2019).

${ }^{1}$ Universidad Central "Marta Abreu” de Las Villas. Cuba. Correo electrónico: adiazj@uclv.cu

${ }^{2}$ Universidad Central “Marta Abreu” de Las Villas. Cuba. Correo electrónico: arleneg@uclv.cu 
The methodology used to carry out the diagnosis is the Document Management and File Management Model of the Transparency and Access to Information Network published in January 2015. The completion of the opinion through this methodology verifies that the status of the Document Management from the perspective of Information Governance at the Universidad Central "Marta Abreu"of Las Villas is at a very elementary level.

Keywords: Document Management, Information Governance, Document Management Model, Universidad Central "Marta Abreu" of Las Villas.

\section{Introducción}

La Gestión Documental se presenta en el siglo XXI como un recurso estratégico de primer orden, mostrando su notable efecto en las esferas económicas, sociales, políticas, tecnológicas y empresariales de cualquier organización. El desarrollo de la Gestión Documental, en un escenario de Gobernanza de la Información, favorece la interacción con otros procesos y actividades informacionales. Tal es el caso de la Gestión de Riesgos y el Control Interno. Esto propicia potenciar el valor de los activos de información y minimizar las dificultades asociadas a la administración documental.

La gestión de documentos es un área de la administración. Puede relacionarse con tareas, procesos y procedimientos para el logro de mayor eficacia en cuanto al uso de los documentos administrativo (Paz, Hernández, Izquierdo, 2016). La seguridad de la información es un factor importante para proteger los activos de una organización. La implementación de un sistema de información evidencia la necesidad de identificar y controlar riesgos. Esto constituye una complejidad adicional al proceso de desarrollo de un sistema de gestión de seguridad de la información. Entender los archivos como elemento fundamental de los sistemas de información, permite establecer medidas de confianza en la toma de decisiones y la transparencia (Riera, Paz y Hernández, 2018).

El control documental, su flujo y vida útil desde su origen hasta su destrucción o almacenamiento permanentemente es otro aspecto que se vincula directamente a este proceso. La gestión documental contempla los procesos de creación, producción, identificación, clasificación y descripción de los documentos como resultado de los métodos administrativos y de negocios en entidades públicas y privadas (Mena, 2019).

En Cuba, el marco regulatorio para la Gestión Documental marca las pautas para el tratamiento de los documentos de archivo en las diversas instituciones. Dentro de este marco destaca la Ley 265 del 2009 (Consejo de Estado, 2009). Esta establece las normas para la actividad archivística en el territorio nacional como parte de la administración pública, la gestión de sus documentos y la administración de sus archivos. Con las disposiciones, esta ley facilita los procesos de Control Interno y Gestión de Riesgos. Asimismo su implementación contribuye a la posterior Gobernanza de la Información como vía o recurso que demanda la iniciativa de gobierno electrónico en Cuba. El Ministerio de Ciencia, Tecnología y Medio Ambiente (CITMA) en conjunto con la Contraloría General de la República son las entidades revisoras y contralorasdel estado de la Gestión Documental en las instituciones cubanas por la relación establecida entre Gestión Documental, Control Interno y Gestión de Riesgos.

La investigación en relación a los diagnósticos enfocados a la Gobernanza de Información no es un área muy prolífica, aunque es necesario destacar un grupo de investigaciones en el ámbito nacional e internacional donde se exponen resultados asociados a los diagnósticos en esta área. En los últimos años se destacan algunas investigaciones realizadas en América Latina como son:

- Gutiérrez (2003) titulado La auditoría de información como herramienta de evaluación y mejoramiento de la gestión de documentos.

- Sierra(2012)bajo el titular Gestión Documental enfocada a procesos: una mirada desde la administración pública distrital.

- Palacios (2014) con el título Diagnóstico de la gestión documental del Archivo General de Bogotá.

- Fernández (2014) bajo el título Gestión documental.

Díaz Jiménez, Asleni; González Pérez. Arlene. (2020). La gestión documental en función de la gobernanza de la información. El caso de la Universidad Central "Marta Abreu" de las Villas. Sapientiae (6) 1, Angola, (Pp. 70-85). DOI: www.doi.org/10.37293/sapientiae61.07 
- Triana, Febles, Mena, González y García(2018) con el título Diagnóstico de los sistemas de gestión documental para desarrollar la gestión del conocimiento.

En el caso de las investigaciones en Cuba, destaca la Facultad de Comunicación de la Universidad de La Habana, como parte de los estudios de pregrado y postgrado, ellas son:

- Font, Ruiz\& Mena (2012) con el título: Diagnóstico sobre la gestión documental y de archivos en la Universidad Central Marta Abreu de las Villas. Cuba: Caso de estudio.

- Ruiz\& Bodes (2014) con La Gestión Documental ysu Impacto en el Sector Empresarial Cubano.

- Arencibia (2015) con el título: Propuesta de herramientas de Gestión Documental para el Control Interno de la Facultad de Comunicación de la Universidad de la Habana.

- Puerta (2016) con la investigación titulada Diagnóstico del estado de la Gestión Documental en la Universidad de La Habana. Este constituye un referente para la estructuración y determinación de los indicadores necesarios para determinar el estado de la Gestión Documental en la Universidad Central "Marta Abreu" de Las Villas.

- Ríos (2016) bajo el título Diagnóstico de la Gestión Documental en los Órganos de la Administración Central del Estado Cubano. Este presenta un enfoque de la Gestión Documental en virtud de la utilidad de dicho proceso para la Gobernanza de la Información y la Gestión de Riesgos.

Es necesario que las instituciones conozcan la importancia de imbricar la Gestión Documental y la Gobernanza de Información, elementos que no deben ser tratados de manera aislada, debido a sus puntos de encuentro e importancia. La Universidad Central "Marta Abreu" de Las Villas (UCLV) nunca antes había sido sometida a un diagnóstico en Gestión Documental en función de la Gobernanza de la Información. La misma enfrenta múltiples problemáticas asociadas a este ámbito, como son la inadecuada gestión de los documentos como elementos de prueba y evidencia de sus funciones. Es por ello que se encuentra expuesta a numerosos riesgos y fisuras en el origen, tratamiento, conservación y recuperación de la documentación proveniente de su administración periódica. En correspondencia definimos como objetivo: diagnosticar el estado de la Gestión Documental en función de la Gobernanza de la Información en la UCLV.

La investigación ofrece una panorámica del Modelo de Gestión de Documentos y Administración de Archivos (MGD) para la Red de Transparencia y Acceso a la Información (RTA)utilizada para la realización del diagnóstico. Posteriormente se exponen la población y muestra utilizadas en el estudio. Finalmente se exhiben los principales resultados teniendo en cuenta los Niveles que establece el Modelo para el caso de los diagnósticos: Nivel Inicial, Intermedio y Avanzado.

\section{Métodos}

La investigación clasifica como una investigación aplicada, pues los fundamentos teóricos y metodológicos sirven de apoyo a la conformación de un resultado, el cual pretende resolver un problema real. Sirve como base para el diseño de posibles estrategias para el mejoramiento de la gestión documental en la UCLV y de problemáticas generales relacionadas con la identificación, tratamiento y disposición de los documentos archivísticos en la entidad.

Utiliza para la realización del diagnóstico el Modelo de Gestión de Documentos y Administración de Archivos (MGD) para la Red de Transparencia y Acceso a la Información (RTA) (Franco y Pérez, 2015). El mismo se conformó con toda la información recopilada mediante los cuestionarios realizados sobre la temática en cada uno de los países implicados en la RTA: Brasil, Colombia, Chile, Ecuador, México, El Salvador, Guatemala, Perú, Santa Fe (Argentina) y Uruguay.

El MGD para la RTA está basado en buenas prácticas internacionales y en el respeto por la diversidad de políticas archivísticas, nacionales y regionales de los países integrantes en la Red, así como de las normas, legislación y reglamentos vigentes en los respectivos ordenamientos jurídicos nacionales. 
Gracias a este esfuerzo se han elaborado una serie de documentos que forman parte del Modelo.El MGD está compuesto por:

- 1 Documento Marco, explicativo del Modelo.

- 8 Guías de Implementación, cuatro de ellas políticas o gerenciales y cuatro de procesos u operacionales.

- 26 Directrices que dependen de las Guías de Implementación.

- 1 anexo con el Informe de diagnóstico. Resultados de las encuestas.

- 1 Anexo con un Cuestionario de Autoevaluación o check-list que permita a cada archivo u organización conocer su nivel en cada uno de los procesos.

- 1 Anexo con un Glosario general, en el que se recogen los términos utilizados en los documentos, lo que facilita su consulta.

Dicho Modelose afianza como una Metodología adecuada para la realización de diagnósticos en Gestión Documental, debido a su amplio enfoque y la inserción de la perspectiva de la Gobernanza de Información.En el caso del presente estudio solo se tomó como referencia la técnica de cuestionario de Autoevaluación o check-list contenida en el Modelo expuesto anteriormente. El mismo permitió conocer el estado en que se encuentran los procesos técnicos documentales en cada una de las dependencias analizadas.

El Cuestionario de Autoevaluación o check-list se encuentra conformado por los niveles inicial, intermedio y avanzado con un total de 868 preguntas.Estos niveles se encuentran compuestos por los ítems o secciones: Política de gestión de documentos y archivo, Gobierno abierto y trasparencia, Administración electrónica, Valoración,Control intelectual y representación, Control de acceso, Control físico y conservación y Servicios. Aunque poseen los mismos ítems el número de preguntas y la profundidad de las mismas varía, esto se debe al objetivo que persigue cada nivel. El nivel inicial va encaminado a conocer el dominio técnico y los procederes archivísticos que deben realizarse con la documentación en formato tradicional y sus responsables. Para ello realiza un mayor énfasis en el Control de Acceso (19 preguntas) y en los Servicios (42 preguntas).

El nivel intermedio, por su parte, está orientado a la administración electrónica de los documento, acentuandoen procesos medulares como: la Política de de gestión de documentos y archivos (80 preguntas), el Gobierno abierto y transparencia (38 preguntas), la Administración electrónica (44 preguntas) y la Valoración (67 preguntas).

Mientras que, el nivel avanzado se enfoca más en aquellos procesos que se deben generar por parte de la alta administración para lograr una óptima gestión documental, destacando el papel de la Política de gestión documental y archivos (66 preguntas), el Gobierno abierto y la transparencia (35 preguntas), la Valoración (63 preguntas) y el Control físico y la conservación (35 preguntas). Los respectivos niveles están estructurados cada uno con ocho secciones y sus respectivas preguntas, cuyo número no es coincidente (Ver Tabla 1). En dichas secciones se interrelacionan la Gestión Documental con la Gobernanza de la Información.

Tabla 1. Descomposición del cuestionario MGD, de acuerdo a la cantidad de preguntas presentadas en sus niveles.

\begin{tabular}{|c|c|c|c|}
\hline Items & $\begin{array}{c}\text { Número de } \\
\text { Preguntas Nivel } \\
\text { Inicial }\end{array}$ & $\begin{array}{c}\text { Número de } \\
\text { Preguntas Nivel } \\
\text { Intermedio }\end{array}$ & $\begin{array}{c}\text { Número de } \\
\text { Preguntas Nivel } \\
\text { Avanzado }\end{array}$ \\
\hline $\begin{array}{c}\text { Política de gestión de documentos } \\
\text { y archivos }\end{array}$ & 47 & 80 & 66 \\
\hline Gobierno abierto y transparencia & 16 & 38 & 35 \\
\hline Administración electrónica & 41 & 44 & 26 \\
\hline Valoración & 55 & 67 & 21 \\
\hline $\begin{array}{c}\text { Control intelectual y } \\
\text { representación }\end{array}$ & 20 & 24 & 12 \\
\hline Control de acceso & 19 & 12 & \\
\hline
\end{tabular}

Díaz Jiménez, Asleni; González Pérez. Arlene. (2020). La gestión documental en función de la gobernanza de la información. El caso de la Universidad Central "Marta Abreu" de las Villas. Sapientiae (6) 1, Angola, (Pp. 70-85). DOI: www.doi.org/10.37293/sapientiae61.07 


\begin{tabular}{|c|c|c|c|}
\hline Control físico y conservación & 24 & 29 & 35 \\
\hline Servicios & 42 & 28 & 24 \\
\hline Total de preguntas por Niveles & $\mathbf{2 6 4}$ & $\mathbf{3 2 2}$ & $\mathbf{2 8 2}$ \\
\hline Total Final & \multicolumn{3}{|c|}{} \\
\hline
\end{tabular}

Fuente: elaboración propia.

Para ello se determinó como Población: lostrabajadores de la UCLV, constituida por:

- La alta dirección, responsable de designar un representante específico líder en el establecimiento, implementación y mantenimiento del sistema.

- Los profesionales de las tecnologías de la información y los administradores de sistemas.

- Técnico de Gestión Documental y archivos, profesionales capacitados para el desarrollo de sus funciones en cualquiera de los procesos archivísticos.

La Muestra,se seleccionó de acuerdo con el muestreo cualitativo. Se establece como mixta, por conveniencia y de voluntarios (Hernández, 2010). Tomando como criterio de selección aquellas dependencias donde existía alguna incidencia y tendencia a la colaboración, acceso y participación en el presente estudio. Se seleccionó una persona para cada nivel del cuestionario, perteneciendo cada una de ellas a una dependencia de la UCLV donde se materializa una de las cinco Áreas de Resultados Clave (ARC). Por ello, en cada ARC fueron seleccionadastres personas de dichas dependencias (Ver Tabla 2). Esto no confirma en el caso de las ARC uno de formación de pregrado y la dos de formación de posgrado, en una misma dependencia, entiéndase, una facultad, coexisten ambas ARC.

Tabla 2. Segmentación de la muestra de acuerdo con las dependencias y ARC, así como los niveles del cuestionario MGD.

\begin{tabular}{|c|c|c|c|}
\hline $\begin{array}{l}\text { Niveles del Instrumento } \\
\text { (Cuestionario MGD) }\end{array}$ & ARC/Dependencia & Implicados & $\begin{array}{c}\text { Total de } \\
\text { encuestados por } \\
\text { niveles }\end{array}$ \\
\hline Inicial & $\begin{array}{l}1 \text { y } 2 / \text { Facultad de } \\
\text { Matemática, Física y } \\
\text { Computación (MFC) } \\
\text { 3/Departamento de } \\
\text { Proyecto } \\
\text { 4/Departamento de } \\
\text { Recursos Humanos } \\
\text { 5/DOPA }\end{array}$ & $\begin{array}{l}\text { Especialista en } \\
\text { Gestión } \\
\text { Documental }\end{array}$ & cuatro personas \\
\hline Intermedio & $\begin{array}{l}1 \text { y } 2 / \text { Facultad de } \\
\text { Matemática, Física y } \\
\text { Computación (MFC) } \\
\text { 3/Departamento de } \\
\text { Proyecto } \\
\text { 4/Departamento de } \\
\text { Recursos Humanos } \\
\text { 5/DOPA }\end{array}$ & $\begin{array}{l}\text { Administradores } \\
\text { de Red }\end{array}$ & cuatro personas \\
\hline Avanzado & $\begin{array}{l}1 \text { y } 2 / \text { Facultad de } \\
\text { Matemática, Física y } \\
\text { Computación (MFC) } \\
\text { 3/Departamento de } \\
\text { Proyecto } \\
\text { 4/Departamento de } \\
\text { Recursos Humanos } \\
\text { 5/DOPA }\end{array}$ & $\begin{array}{l}\text { Director (tres } \\
\text { personas) }\end{array}$ & \multirow[t]{2}{*}{ cuatro personas } \\
\hline \multicolumn{2}{|c|}{ Total de Encuestados } & 12 personas & \\
\hline
\end{tabular}

Fuente: elaboración propia. 


\section{Resultados \\ Estado actual de la Gestión Documental en función de la Gobernanza de la Información en la UCLV}

Los diferentes niveles (inicial, intermedio y avanzado) hacen alusión, mediante los ítems, a los aspectos que deben contemplar la institución según las áreas y responsables de la documentación. Los niveles responden a los principios de responsabilidad, transparencia, integridad, protección, cumplimiento, disponibilidad, retención y disposición planteados por ARMA Internacional (2018). Los gráficos muestranla presencia de todos los ítems correspondientes a cada nivel, según establece el cuestionario aplicado.

\section{Nivel Inicial}

El nivel inicial hace énfasis en los diferentes procesos y subprocesos asociados a las actividades, reglamentaciones, normativas y a las diversas posturas a asumir por la institución para el uso adecuado de su documentación para usuarios internos y externos. Este nivel engloba los elementos necesarios a tener en cuenta en las diferentes fases del tratamiento archivístico desde el control de la creación documental hasta la difusión (Ver Gráfico 1).

Gráfico 1. Nivel Inicial del estado de la Gestión Documental en función de la Gobernanza de la Información en la UCLV.

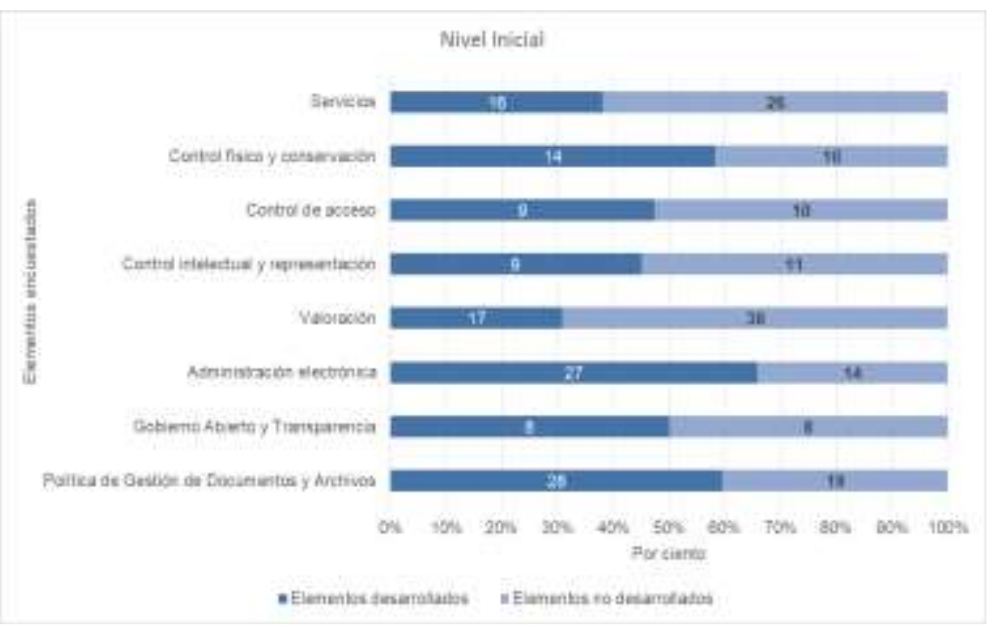

Fuente: elaboración propia.

El ítem Servicios con un total de 42 preguntas, sólo fueron contestadas favorablemente 16 de ellas. Esto representa un 38\% del total. El resultado responde al desconocimiento por parte de los archiveros de las potencialidades informativas que posee su fondo documental. Por ende, se limitan a brindar los servicios, sólo a los usuarios internos. El aspecto anterior es característico de los sistemas institucionales de archivo en los niveles primarios dentro del siclo de vida documental (Gutiérrez, 2003). Los archivos de gestión e intermedios generalmente solo prestan sus servicios a los usuarios internos de instituciones (Triana et al, 2018). El préstamo interno es uno de los aspectos que tipifican los archivos de gestión, cuya finalidad son fundamentalmente las dependencias internas instituciones (Sobhani et al, 2011).

El Control físico y la conservación poseen 24 interrogantes, de ellas 14 fueron respondidas favorablemente para un 58\%. Lo anterior evidencia que aunque se realiza un control sistemático a la documentación, aún se deben afianzar aspectos como la conservación. El Control físico ejemplifica el trabajo realizado por parte de los especialistas, aunque aún es insuficiente. En el mismo investigan las necesidades y recursos del organismo para concretar los requisitos de custodia. Analizando los factores de riesgo asociados a los documentos y al personal del centro. 
La conservación de los documentos en instalaciones debidamente autorizadas es deficiente debido a la inexistencia de un Programa de Conservación Preventiva, ni un Plan de Contingencias.Dicho elemento es poco tratado en la entidad y por ende el conocimiento sobre el mismo y las acciones enfocadas en el mantenimiento de los documentos es muy precario. Los resultados obtenidos difieren de las experiencias desarrolladas en estudios en otros contextos organizacionales. A criterio de Paz y del Sol (2017): "uno de los aspectos esenciales para el desarrollo de una adecuada gestión documental es el almacenamiento y custodia adecuada de la documentación" (p. 91). Las condiciones adecuadas de almacenamiento evitan el deterioro de las series documentales generadas por las organizaciones (Real y Bateoja, 2018).

El Control de acceso de 19 interrogantes, nueve fueron contestadas afirmativamente, representando un 47\%. Ello evidencia el control del acceso a la documentación, aunque afirman los encuestados que se realiza de manera empírica y existe ausencia de un reglamento o normativa institucional regulando este aspecto.

El Control intelectual y la representación con un total de 20 incógnitas, presenta solo nueve de ellas respondidas positivamente, representando un $45 \%$ del total. Tal porciento demuestra el desconocimiento por parte del personal responsable de esta actividad. La ausencia de la identificación de las estructuras, funciones básicas, así como de los documentos generados por estas funciones dentro de la organización es un elemento muy importante que no se realiza en su totalidad. No se le otorga una verdadera jerarquía a la identificación de los tipos documentales, siendo esta actividad un factor de éxito para las organizaciones. Cuando se obtienen estos resultados se evidencia un alto riesgo para las organizaciones (Paredes y Escobar, 2014). El aspecto anterior supone que deban crearse herramientas de control documental que permitan una adecuada gestión de documentos (Cabezas, 2014).

La Valoración, un ítem con 55 preguntas, presenta aproximadamente un 31\%, con sólo 17 respuestas favorables. Este proceso no se realiza con toda la regularidad, eficiencia y seriedad requeridas. Evidencia de ello es la usencia de la Comisión de Valoración Documental, el desconocimiento del proceder y de los diferentes modelos que deben regular esta actividad. Siendo así el ítem con más bajo resultado en este nivel.Sobre este tipo de resultados Castillo y Ravelo (2017) establecen que "el proceso de valoración documental, cuando no se realiza adecuadamente, pone en riesgo a las organizaciones las cuales pueden deshacerse de documentación relevante cuya pérdida deviene en perjuicios" (p. 275). La no realización de la valoración documental es muestra de la ausencia de herramientas en la gestión de documentos como la tabla de retención documental (Borja et al, 2018)

La Administración electrónica presenta 41 interrogantes, de ellas 27 respondidas positivamente, representando un 65\%. Ello evidencia el insuficiente trabajo que aún se realiza en este ítem, destacando la baja interoperabilidad técnica y organizativa lograda institucionalmente. Los resultados obtenidos se relacionan con el ítem anterior como consecuencia de la inadecuada valoración documental (Montserrat, 2008). La óptima administración de los documentos electrónicos y las reglamentaciones asociadas a la seguridad de esta información son aún mecanismos pendientes por diseñar e implementar. A pesar del criterio anterior, Cabezas (2014)establece que "el tratamiento de los documentos electrónicos debe ser igual de relevante para las organizaciones como el tratamiento a la documentación tradicional” (p. 4).

El Gobierno abierto y la transparencia está compuesto con un total de 16 preguntas, de ellas, sólo la mitad: ocho, fueron contestadas favorablemente, representando un $50 \%$. En este apartado nose elaboran y diseñan cuadros de clasificación, ni otros instrumentos de descripción documental adecuados en dependencia del tipo de usuario, ello atenta contra la difusión informativa y la trasparencia informacional. Existe una clasificación parcial de los requisitos legales y operativos que afectan a la organización.

Cabe destacar la implementación de las restricciones y procedimientos de control en el uso a los documentos que son considerados de acceso restringido, aspecto tratado con mucha rigurosidad

Díaz Jiménez, Asleni; González Pérez. Arlene. (2020). La gestión documental en función de la gobernanza de la información. El caso de la Universidad Central "Marta Abreu" de las Villas. Sapientiae (6) 1, Angola, (Pp. 70-85). DOI: www.doi.org/10.37293/sapientiae61.07 
siendo este aspecto el mejor desarrollado. Los resultados obtenidos en el ítem repercuten de forma negativa en la organización. La ausencia de herramientas funcionales y operativas en la gestión documental constituyen una debilidad para las organizaciones (Sour, 2017).

La Política de gestión de documentos y archivo comprende un total de 47 preguntas, de etas, sólo 28 resultaron positivas representando un 59\%. Ello evidencia que la Política no existe, aunque sí existe conocimientoque la misma debe ser promovida y liderada desde las más altas autoridades de la organización. Por otra parte se realizan estrategias para tributar los recursos indispensables para el buen desarrollo de las labores en gestión documental. No existe un diseñode los modelos gráficos de las diversas unidades que ostenta la organización, ni seidentifican los requisitos para el Sistema de Gestión de Documentos que existe, pero funciona deficientemente.

Aunque la mayor parte de los ítems fueron positivos, no puede considerarse que el sistema sea fiable y adecuando en cuanto a la gestión de documentos. Las políticas de gestión documental contienen aspectos relativos a la creación, procesamiento, acceso, dirección, intercambio, distribución, seguridad, flujos y otras actividades relacionadas con la información (Sebastián y Cardama, 2016). La elaboración y diseño de políticas de gestión documental forman parte de la gerencia estratégica, por lo que resulta imprescindible para las organizaciones (Paredes y Escobar, 2014).

En el nivel inicialse aprecia una carencia de buenas prácticas en el ámbito de la gestión documental, aunque no es una generalidad. Asimismo, en el desarrollo de instrumentos de control y descripción documental. No existe conocimiento del marco regulatorio imperante en los archivos, ni las regulaciones pertenecientes al Ministerio de Educación Superior en Cuba por parte de los archiveros. También la infraestructura presenta varias problemáticas, aunque preliminarmente las adecuaciones de un inmueble de este tipo (edificación para archivo) son conocidas, pero no existe una concientización, ni recursos materiales para esta labor.Muchas de las prácticas se realizan empíricamente, no por esto incorrectamente. Ello implica que una vezque el archivero que se encuentra a cargo se retira de su puesto, no se encuentren instrumentos que faciliten el conocimiento sobre el trabajo con la documentación.

\section{Nivel Intermedio}

El nivel intermedio hace énfasis a la documentación en formato digital. Aborda los elementos esenciales que dan soporte a lo conocido como Gobierno abierto y transparencia. Entre ellos se destacan la normalización de los procesos en gestión documental, la identificación de requisitos para el Sistema y la trasparencia activa y los datos abiertos, para así logar la óptima administración electrónica de los documentos en este formato (Ver Gráfico 2).

Gráfico 2. Nivel Intermediodel estado de la Gestión Documental en función de la Gobernanza de la Información en la UCLV.

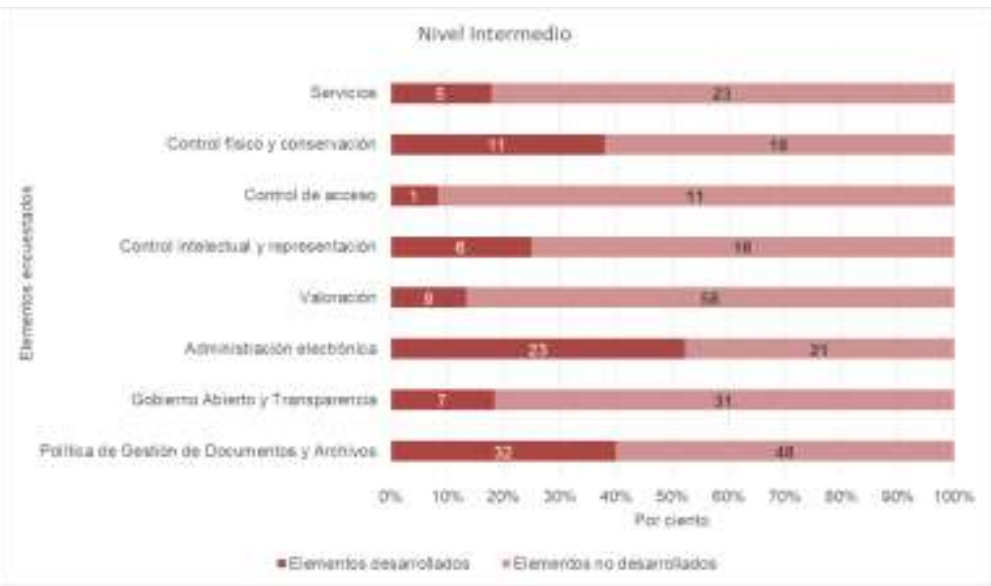

Fuente: elaboración propia.

Díaz Jiménez, Asleni; González Pérez. Arlene. (2020). La gestión documental en función de la gobernanza de la información. El caso de la Universidad Central "Marta Abreu" de las Villas. Sapientiae (6) 1, Angola, (Pp. 70-85). DOI: www.doi.org/10.37293/sapientiae61.07 
El ítem Servicios con un total de 28 interrogantes, sólo posee respondidas satisfactoriamente cinco de ellas para un $17 \%$. Tan bajo resultado es consecuencia de la poca relación que se establece entre las temáticas de disposición y uso de los documentos. No existen servicios de atención continuada a la administración productora, ni a los usuarios externos. El personal de servicio de atención al público no se encuentra especializado en atención a usuarios, además se debe destacar la ausencia, dentro de la plantilla de la unidad de archivo, de las funciones relativas a la actividad de difusión.Aunque se incorporan indicadores de atención al público en los procesos y cuadros de mando integral de la organización, en la medida del análisis de posibles estrategias en todas las dependencias. Esto les permite analizar posibles estrategias de difusión y promoción para acercar los documentos a los usuarios.

Los servicios en el ámbito de la archivística constituyen un conjunto de actividades enfocadas a satisfacer necesidades de información de los usuarios y clientes del sistema de gestión archivístico (Fernández, 2012). Los servicios constituyen el ofrecimiento de las instituciones a sus usuarios, por lo que resultan indispensable en las organizaciones (Nicandro, 2015). El diseño e implementación adecuado de servicios de información archivístico permite a los usuarios potenciales del sistema tener las herramientas adecuadas para la toma de decisiones (Moyano, 2015).

El Control físico y la conservación poseen 29 preguntas, respondidas favorablemente 11, para un $37 \%$. Esto se debe a la baja identificación de los documentos generados en la organización, de ahí la escasa o inadecuada clasificación que se le realiza a los mismos. Por tanto, los instrumentos de organización documental son prácticamente nulos, aunque se pueden encontrar Cuadros de Clasificación Documental en varias dependencias. Aunque su calidad y exhaustividad pueden ser muy cuestionables.

La identificación documental es uno de los procesos primarios para garantizar una adecuada gestión de documentos. La existencia de cuadros de clasificación en las organizaciones favorece un adecuado tratamiento de la documentación generada. Lo anterior es consecuencia de la necesidad de herramientas funcionales que permitan un funcionamiento óptimo de los sistemas de gestión documental (Giménez, 2012).

El control de acceso con 12 interrogantes, sólo presenta un ítem favorable, representando un 8,3\%. Este fenómeno responde al escasísimo control en cuanto al acceso a la documentación en formato electrónico. Existe ausencia de solicitudes de acceso a los documentos públicos. El aspecto anterior puede ser mejorado a partir de la implementación de un sistema de gestión documental automatizado. Se deberá utilizar un registro o sistema de tramitación automatizado que controla el flujo de tareas, donde se crea un conjunto de permisos de usuario de la organización o un registro propio. En el registro se pueden establecer las necesidades de acceso de las distintas áreas funcionales de la organización y así analizar las restricciones de acceso y requisitos de seguridad de los documentos (Castillo, 2016). Aunque en la institución analizada no existe un sistema automatizado, si pudo identificarse una propuesta en el estudio desarrollado por Font,Ruiz y Mena (2012).

El Control intelectual y la representación presentan 24 incógnitas, sólo seis respondidas afirmativamente, para un $25 \%$. Este bajo resultado se debe al escaso trabajo en el programa de custodia documental, donde no se delimitan claramente los responsables en la custodia, trasferencias o eliminación de los documentos. Por otra parte, no existe documentado una justificación o difusión de las decisiones sobre la conservación de los documentos. No se exhiben garantías sobre la seguridad de los documentos en las instalaciones de depósito, ni de la implementación y mantenimiento de la gestión del biodeterioro. Finalmente se hace necesario enfatizar que no existe un Plan de Contingencias como culminación de la fase control.

El control como proceso dentro de la gestión de documentos, es el que garantiza la integridad y adecuada custodia de la documentación (Briano, 2014). Dentro del proceso deberán diseñarse

Díaz Jiménez, Asleni; González Pérez. Arlene. (2020). La gestión documental en función de la gobernanza de la información. El caso de la Universidad Central "Marta Abreu" de las Villas. Sapientiae (6) 1, Angola, (Pp. 70-85). DOI: www.doi.org/10.37293/sapientiae61.07 
políticas que prevengan daños a los que pueden estar sometidos los documentos. Dentro de las políticas más recurrentes se encuentran 1) las enfocadas en el almacenamiento, conservación y preservación de documentos y 2) las que establecen planes de prevención contra catástrofes naturales o provocadas por el hombre (Campilloy Cabrera, 2016).

La Valoración documental con 67 interrogantes, destaca por presentar nueve preguntas respondidas satisfactoriamente, para un 15\%. En este proceso se ausenta el análisis de estas series documentales digitales, las funciones, organismos productores, necesidades sociales y administrativas de la organización. Por ende no se encuentra identificado el personal encargado de llevar a cabo las transferencias y la eliminación dentro de la organización. El mismo no evidencia la presencia de estudios de los valores documentales, no documentan los criterios de valoración ni comunican las decisiones y dictámenes a todas las partes implicadas, por tanto estamos en presencia de una fase tan necesaria en el entono digital y que no se realiza. Cabe destacar que los plazos de prescripción de la documentación sí son identificados con el análisis del trámite administrativo, plazos legales y jurídicos, según la normativa vigente, aunque no son funcionales dentro del Sistema. Resultados similares fueron obtenidos por Cabezas (2014), donde se establece que por lo general los sistemas de gestión electrónica de documentos son descuidados.

La Administración electrónica con 44 interrogantes, fueron respondidas 23, para un 52\%. Esto responde a la existencia de la conectividad, la comunicación existente entre los equipos, así como la generalización del acceso a Internet. Aunque aún se debe potenciar esta conectividad al igual que el acceso a Internet para potenciar los servicios y acercarlos a los ciudadanos. Se deben armonizar los dominios de información y el uso de los datos abiertos (Mena y Dorado, 2009). Se debe fomentar el liderazgo institucional, ya que es una necesidad básica, así como la explotación segura y controlada de la infraestructura, con la debida supervisión y registro de incidencias (Paredes y Escobar, 2014). Por otra parte, se hace necesario impulsar la integridad de la seguridad de la información en los sistemas y considerar la firma electrónica como un elemento del documento electrónico que, además del carácter identificativo, aporta integridad y evita su rechazo (Ramírez, 2015).

El Gobierno abierto y la transparencia presentan un total de 38 interrogantes, de ellas, sólo siete respondidas favorablemente, para un 18\%. Este resultado tan bajo se debe a la inexistencia de una política o normativa sobre los documentos públicos, donde se declaren los principios relativos a su acceso. Tampoco existe una estrategia de trasparencia activa y datos abiertos que permita la apertura por defecto de la información del archivo y su publicación en la Red, así como las razones de exclusión para aquella información que lo requiera. A lo anterior se le suma la inexistencia de mecanismos de participación y colaboración que diseñen una estrategia para la puesta a disposición de la información de la organización para su reutilización permitiendo la creación de un espacio web para ubicar los recursos reutilizables y la información sobre los mismos, así como las herramientas open data, de consulta y localización de los datos pertinentes.

Debe potenciarse la creación de políticas públicas para facilitar la transparencia administrativa a partir del acceso a la información. La normativa y leyes, la transparencia y la rendición de cuentas fortalecen la estructura y perspectiva de gobierno abierto de gobierno (Ramírez, 2015). Alguna de las variables que inciden en el nivel de transparencia se localizan en las dimensiones políticas, sociales y económicas (Núñez y Oneto, 2014). La elaboración de índices de divulgación es una de las herramientas más utilizada para medir la transparencia corporativa (Gandía, 2008). Por lo que deben crearse estrategias que permita visibilizar el trabajo y la gestión archivística organizacional. La Política de Gestión de Documentos y Archivos con 80 interrogantes, presenta 32 respondidas positivamente, para un $40 \%$. Se hace necesario destacar que este ítem es el de más preguntas dentro de todo el instrumento, ello demuestra la importancia concedida a la utilización de Políticas en cuando a documentación digital se trata. Este porciento bajo responde a la necesidad de estandarizar desde la dirección institucional los procesos relativos a la gestión documental. 
El estudio previo de la organización, su Matriz DAFO y la identificación de las estrategias para cumplir con los requisitos en gestión documental son tácticas que no se realizan con mucha frecuencia en la institución. Mientras la normalización de los procesos, la elaboración de fichasde proceso,el mapeo de los mismos, la asignación de roles, responsabilidades y competencias necesarias en materia de gestión de documentos dentro de la organización son elementos no tratados con frecuencia. Se hace necesario trazar una Política que incluya estos documentos y los integre coherentemente dentro del Sistemade forma conjunta (Prado y Navarro, 2016).

En el presente nivel se encuentran las mayores problemáticas asociadas al tratamiento de los documentos electrónicos, destacando el análisis incompleto de las series documentales generadas por la institución y su posterior tratamiento como documentos archivísticos. Figuran problemáticas como el control al acceso y el establecimiento de plazos de conservación. Destaca la necesidad expedita de la adopción de una Política que permita poner a disposición la información de la organización para su reutilización permitiendo la creación de un espacio web para ubicar los recursos reutilizables y la información sobre los mismos, así como las herramientas open data, de consulta y localización de los datos pertinentes.

\section{Nivel Avanzado}

El nivel avanzado centra su atención en el conocimiento general por parte de la alta dirección de la institución en materias relacionadas con la gestión documental. El conocimiento de los procesos que componen la gestión documental proporciona institucionalmente el desarrollo de esta rama y apoyan directamente el desarrollo institucional. El conocimiento profundo de los mismos por la alta dirección, hace de ellos unidades funcionales, articuladas y coherentes (Ver Gráfico 3).

Gráfico 3. Nivel Avanzado del estado de la Gestión Documental en función de la Gobernanza de la Información en la UCLV.

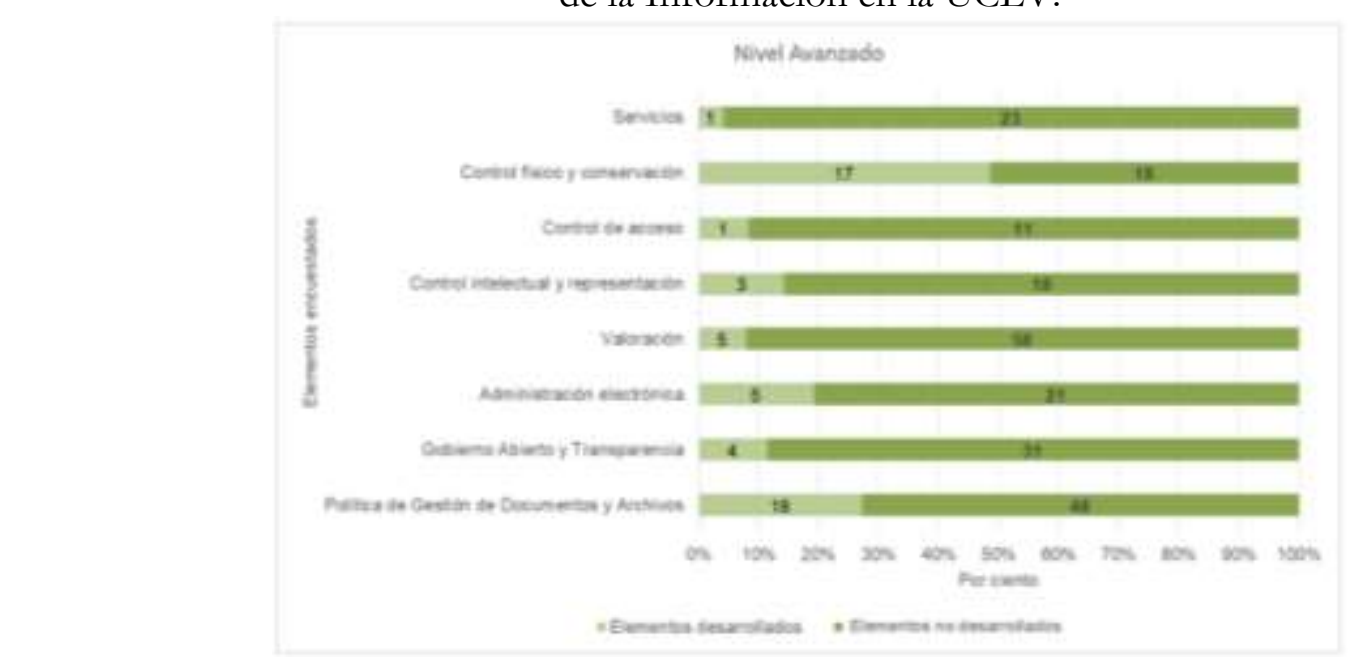

Fuente: elaboración propia.

El ítem Servicios de un total 24 preguntas, fueron contestadas positivamente sólo una, para un $4,1 \%$. Esto responde al desconocimiento, por parte de la alta dirección de la institución, de las potencialidades informativas que poseen los documentos para brindar servicios de información. A la inexistencia de un servicio de atención continuada a la administración productora y a su respectivo sistema integral de control de documentos en préstamo administrativo para toda la organización. Tampoco existen servicios de atención continuada a usuarios externos y por ende tampoco encuestas de satisfacción que muestre información sobre el funcionamiento de estos servicios.

Es necesario el diseño de una política integral que contemple todas las actuaciones en materia de atención a usuarios. Las universidades como sujetos productores de documentos,deben ofrecer 
servicios a usuarios externos. Dentro de la tipología de servicios de información que deben ofrecer se encuentran los de facilitación social, reproducción de documentos, de referencia (telefónica, virtual y cara a cara) (Mera, Paz y Hernández, 2016).

El Control físico y la conservación muestra un mejor estado, de 35 interrogantes, 17 fueron positivas, representando el 48\%. El mismo representa el interés institucional por la preservación de sus documentos. Debido a esto, se trata de cumplir, según el nivel de recursos con que se cuenta, con el adecuado mantenimiento de los depósitos donde se almacenan los documentos, realizando inspecciones regularmente. La institución traza estrategias y herramientas potenciando la durabilidad de los soportes y la obsolescencia tecnológica. Son activadas las medidas, protocolos planificados y diseñados tras los desastres con el objetivo de minimizar su impacto sobre los documentos.

Se debe enfatizar en el trabajo con la conservación preventiva y en la separación de las instalaciones de depósito de documentos de las demás zonas de trabajo de la organización. La prevención y el control ambiental, aunque no es un punto totalmente descuidado, sí deben afianzar su trabajo en el aseguramiento de que los sistemas de preservación y control empleados conserven los documentos electrónicos y sus metadatos asociados, así como la activación de las medidas y protocolos planificados y diseñados tras los desastres con el objetivo de minimizar el impacto (Gauchi, 2015).

El Control de acceso con 12 preguntas, sólo una de ellas fue contestada satisfactoriamente representando un 8,3\%. Esto se debe al escaso análisis de las restricciones de acceso y requisitos de seguridad de los documentos. Se debe establecer un procedimiento de control de acceso, tanto a la documentación en formato tradicional como electrónico conforme a la Norma ISO 15489. Asignación de controles fundamentada en un análisis de riesgos detallado para cada serie documental, así como de los metadatos para la seguridad apropiados a cada clase de documentos. Se debe suscribiruna tabla de acceso y seguridad con los mecanismos para su revisión periódica (Bustelo, 2011).

El Control intelectual y la representación con un total de 21 interrogantes, fueron respondidas favorablemente tres, para un 14,2\%. Ello se evidencia en el poco trabajo que se desempeña en la identificación de la organización y sus documentos, pues se tiene un desconocimiento sobre las incidencias ocasionadas sobre la documentación y sobre las mejoras en la creación de documentos. La clasificación documental, es uno de los procesos que más se realiza, aunque con dificultad, además de ello no se realizan revisiones periódicas de los Cuadro de Clasificación Documentales. Por su parte la descripción documental carece de un Plan de Descripción Archivística o un Manual para su ejecución. La descripción ejecutada, es poca y se realiza empíricamente, según el criterio de los especialistas. En situaciones similares, el autor Ramírez (2015) establece que esnecesaria la adopción de una política archivística en materia de descripción definida para toda la organización o el sistema de archivos.

La Valoración con 63 preguntas, sólo posee cinco respuestas satisfactorias, representando un 7,9\%. Este rublo muestra resultados realmente bajos, teniendo en cuenta su importancia para el manejo de los volúmenes documentales. La institución no le otorga el debido valor y en la misma no se realizan análisis de las series, ni estudios de los valores documentales. Así mismo no son definidos los criterios de valoración, ni son evaluadas propuestas de valoración con sus correspondientes responsables. No se encuentra estructurado el Plan Anual de Transferencias y no se registran los documentos eliminados, ni existe ningún responsable por parte de esta actividad. Los resultados obtenidos a criterio de Real y Bateoja (2018) son muestra de malas prácticas con el tratamiento documental. La ausencia de herramientas no favorece la gestión de documentos de forma óptima. La Administración electrónica cuenta con 26 interrogantes, de ellas sólo cinco fueron contestadas favorablemente para aun 19,2\%. Este valor se corresponde a la baja comprensión por parte de los directivos de la clasificación y la organización como base indispensable para la interoperabilidad. No se trata la interoperabilidad semántica desde un punto de vista técnico, ni idiomático. Por su

Díaz Jiménez, Asleni; González Pérez. Arlene. (2020). La gestión documental en función de la gobernanza de la información. El caso de la Universidad Central "Marta Abreu" de las Villas. Sapientiae (6) 1, Angola, (Pp. 70-85). DOI: www.doi.org/10.37293/sapientiae61.07 
parte, la administración de documentos electrónicos carece de un diseño dentrodel sistema de gestión en el que tengan cabida los metadatos asociados a estos documentos. Debe diseñarse una política de preservación digital donde exista un esquema de metadatos como instrumento que contribuya a garantizar la conservación de los documentos a largo plazo y que permita abrir un marco de interoperabilidad (Nahabetián, 2015).

El Gobierno abierto y la transparencia con un total de 35 preguntas, presenta solamente cuatro contestadas favorablemente, esto representa un 11,4\%.Este comportamiento se debe a la existencia deun sistema de trabajo al más alto nivel que propicia el intercambio y la rendición de cuentas en varios niveles organizacionales, aunque aún es incipiente. Consta la ausencia de una política o norma aprobada al más alto nivel institucional sobre la determinación y el acceso a los documentos públicos. Esta iniciativa posee como base el vínculo indisoluble que debe poseer el fondo documental para la rendición de cuentas y la transparencia administrativa. A nivel institucional, no existe una concientización profunda sobre el uso público de los documentos y la posterior incorporación de las opiniones de los disímiles agentes interesados.

La adopción de una estrategia de trasparencia activa y datos abiertos es un estado deseado que debe antecederle un grupo significativo de tareas y herramientas que aún no se encuentran desarrolladas por la institución. Además de esto, se debe incentivar el liderazgo en el proceso de auditoría y representación de la información, para implementar dichas políticas de transparencia activa y datos abiertos. Una vez creados los canales en las organizaciones se debe potenciar la participación activa de los implicados a través de políticas (Nicandro, 2015).

La Política de gestión de documentos y archivo posee 66 interrogantes, de ellas, 18 contestadas satisfactoriamente, representando un $27 \%$. Esto responde a inexistencia de la Política, aunque existen algunas iniciativas que funcionan dentro del Sistema, las mismas no se encuentran documentadas. A criterio de Paredes y Escobar (2014) para la conformación armónica de esta Política, se debe realizar un análisis de los riesgos que pueden afectar al sistema de gestión de documentos y archivos de la organización. A continuación, realizar el seguimiento y evaluación de los proyectos y actividades existentes, evaluar los resultados y establecer la relación de los procesos y sus actividades con la asignación de competencias y responsabilidades entre el personal implicado en la gestión de documentos. Sobre los aspectos anteriores Moyano (2015) agrega que se debe materializar un documento formal o Modelo de Requisitos en el que se articulan las especificaciones y los requisitos necesarios respecto al mantenimiento, tratamiento y conservación de los documentos en el propio Sistema de Gestión de Documentos. Finalmente se debe elaborar un documento con un sistema de indicadores e identificación de los indicadores más relevantes que conforman el Cuadro de Mando Integral de la organización en materia de gestión de documentos y archivos, que pueda ser acreditable.

En este nivel se aprecia la no existencia de políticas de difusión documental, de trasparencia activa y datos abiertos,elbajo control de acceso y requisitos de seguridad. Se evidencia el poco control sobre los documentos. Aunque cabe destacar por parte de los directivos, el conocimiento sobre el marco regulatorio a manejar y la importancia de determinar las debilidades y amenazas que se deben tener identificadas en cuanto a los documentos.

\section{Conclusiones}

La gestión documental, como proceso clave para el óptimo desarrollo de la gobernanza de la información en las instituciones de la Educación Superior, debe enfatizar en el perfeccionamiento de subprocesos como la captura, mantenimiento, disposición y difusión de los documentos. El nivel inicial muestra los resultados más favorables debido al trabajo mancomunado realizado por los archiveros, aunque corresponde trabajar en aspectos tales como la valoración documental y los servicios, ítems que apoyan directamente al gobierno abierto y la transparencia. Por su parte, el nivel intermedio muestra una escasa ejecución en los procesos técnico-documental asociados a la documentación electrónica, así como el control al acceso y los servicios que puede prestar la misma

Díaz Jiménez, Asleni; González Pérez. Arlene. (2020). La gestión documental en función de la gobernanza de la información. El caso de la Universidad Central "Marta Abreu" de las Villas. Sapientiae (6) 1, Angola, (Pp. 70-85). DOI: www.doi.org/10.37293/sapientiae61.07 
a la administración. Finalmente, el nivel avanzado ejemplifica el escaso conocimiento por parte de los directivos institucionales sobre las temáticas asociadas a la gestión documental y los procedimientos que deben realizarse desde la más alta dirección para potenciar el desarrollo de la misma.

\section{Referencias bibliográficas}

Arencibia, Miguel. (2015). Propuesta de herramientas de Gestión Documental para el Control Interno de la Facultad de Comunicación de la Universidad de la Habana. Alcance. Vol. 3 No. 2 Cuba (Pp. 36-49).

ARMA Internacional. (2018). ARMA. Extraido de: https://www.arma.org/page/igim Consulta: 14/05/19.

Borja, Lucy Katherine; Murillo, Norberto Vicente; Pizarro, Luis Eduardo; Rendón, Deidama Asunción; Meneses, Luis Alberto. (2018). Definición de un Sistema de Gestión Documental en la Universidad de Milagro. Contribuciones a las Ciencias Sociales. Vol. 4Chile (Pp. 1-35).

Briano, Guadalupe del Carmen. (2014). Factores que inciden en una mayor transparencia de gobernanza corporativa en empresas cotizadas latinoamericanas. Revista Mexicana de Economía y Finanzas. Vol. 9 No. 2 México (Pp. 105-124).

Bustelo, Carlota. (2011). Los grandes temas relacionados con la gestión de documentos: desafíos y oportunidades. El profesional de la información. Vol. 20 No. 2 España (Pp. 129-133). DOI: http://dx.doi.org/10.3145/epi.2011.mar.01

Cabezas, Cristian. (2014). Transparencia activa: Gestión de documentos electrónicos y datos en Chile. Serie Bibliotecología y Gestión de Información, Vol. 93Chile (Pp.1-18).

Campillo, Irima; Cabrera, Idalmis María. (2016). Propuesta organizacional de un archivo de gestión para organizaciones empresariales. Ciencias de la Información.Vol. 40 No. 1 Brasil (Pp. 77-84).

Castillo, Jorge; Ravelo, Grettel. (2017). El proceso de valoración documental a la luz de los actuales debates archivísticos. Revista Interamericana de Bibliotecología. Vol. 40 No. 3 Colombia (Pp. 273-283). DOI: http://doi.org/10.17533/udea.rib.v40n3a07

Castillo, Juan Miguel. (2016). La estructuración de sistemas de gestión de documentos y archivos como base para la implementación de un sistema de preservación digital. Cuadernos de Investigaciones de Ciencias de la Información. Vol. 4 No. 5 Brasil (Pp. 55-68). DOI: https://doi.org/10.34295/cuinci.vi1.6

Consejo de Estado. (2009). Decreto-Ley No. 265/2009 "Del Sistema Nacional de Archivos de la República de Cuba". Gaceta Oficial de la República de Cuba. Cuba.

Fernández, Francisco. (2012). Al servicio de la transparencia. El papel de los archiveros y la gestión documental en el acceso a la información pública. Métodos de Información. Vol. 3 No. 5 España (Pp. 153-166). DOI: http://dx.doi.org/10.5557/IIMEI3-N5-153166

Fernández, Luis Daniel. (2014). Gestión documental. Editorial Félix Varela. Cuba.

Font,Odalys; Ruiz, Antonio Ángel y Mena, Mayra Marta. (2012). Diagnóstico sobre la gestión documental y de archivos en la Universidad Central "Marta Abreu" de las Villas. Cuba: Caso de estudio. Revista Española de Documentación Científica. Vol. 35 No. 4 España (Pp. 573-598). DOI: http://doi.org/10.3989/redc.2012.4.883

Franco, Beatriz y Pérez, Ricard. (2015). Modelo de Gestión de Documentos y Administración de Archivos (MGD) para la Red de Transparencia y Acceso a la Información (RTA).Programa EUROsociAL.España. Extraido de:http://mgd.redrta.org/modelo-de-gestion-dedocumentos-y-administracion-de-archivos-para-la-red-de-transparencia-y-acceso-a-lainformacion/mgd/2015-0123/093820. Consulta: 14/05/20 
Gandía, Juán. (2008). Determinants of Interest-Based Corporate Governance Disclosure by Spanish listed Companies. Information Review. Vol. 32 No. 6 Estados Unidos (Pp. 791 817). DOI: https://doi.org/10.1108/14684520810923944

García, Elisa. (2012). Gobernanza de la información.Anuario ThinkEpi. Vol.6 No. 1 España(Pp. 100-103).

Gauchi, Verónica. (2015). Aproximación teórica a la relación entre los términos gestión documental, gestión de información y gestión del conocimiento. Revista Española de Documentación Científica. Vol. 35 No. 4 España (Pp. 531-554). DOI: https://doi.org/10.3989/redc.2012.4.869

Giménez, Vicent. (2012). Acceso de los ciudadanos a los documentos como transparencia de la gestión pública. El Profesional de la Información. Vol. 21 No. 5 España (Pp. 504-508). DOI: http://dx.doi.org/10.3145/epi.2012.sep.09

Gutiérrez, Liliana (2003). La auditoría de información como herramienta de evaluación y mejoramiento de la gestión de documentos. Biblios.Vol. 4 No. 16 España (Pp. 14-22.)

Hernández, Roberto. (2010). Metodología de la investigación.Editorial The McGraw-Hill. México.

Mena, Mayra Marta; Dorado, Yanara. (2009). Evolución de la ciencia archivística. ACIMED. Vol. 20 No. 1 Cuba (Pp. 9-19)

Mena, Mayra Marta; García, Luis Alberto (2019). Eficiencia administrativa y gestión de riesgos. Escenario actual de las políticas de gestión documental. Pedagogía y Sociedad. Vol. 22 No. 55 Cuba (Pp. 213-235).

Mera, Rosa Marina; Paz, Luis Ernesto y Hernández, Eduardo Alejandro. (2016). VER-SIÓN: servicio de información para la inclusión educativa de estudiantes con diversidad funcional visual en la Universidad Técnica de Manabí, Ecuador. Informatio. Revista del Instituto de Información de la Facultad de Información y Comunicación. Vol. 21 No. 2 Uruguay (Pp. 101-120)

Montserrat, Alsina (2008). Metodología para la implantación de la gestión del conocimiento a partir de técnicas documentales. Scire. Vol. 14 No. 1, España (Pp. 43-61).

Moyano, Julián. (2015). Gestión documental en un marco de transparencia y la reutilización de la información. Revista Catalana de Archivística. Vol. 2 No. 14, España (Pp. 45-61).

Nahabetián, Laura. (2015). Protección de datos y gestión documental: decálogo ampliado para la sociedad de la información. Revista de la Facultad de Derecho. Vol. 39, Uruguay (Pp. 199-225).

Nicandro, César. (2015). ¿Qué es (y que no es) gobierno abierto? Una discusión conceptual. Eunomia. Revista en Cultura de la Legalidad.Vol. 8, Colombia (Pp. 37-53).

Núñez, Gilberto;Oneto, Gaspar. (2014). La gobernanza corporativa y la emisión de deuda corporativa en Chile y el Perú.Editorial Comisión Económica para América Latina y el Caribe. Chile.

Palacios, Jorge. (2014). Diagnóstico de Gestión Documental. Secretaria General - Grupo de Archivo y Gestión Documental. Colombia.

Paredes, Ana Judith; Escobar, Lisa. (2014). Lineamientos estratégicos para la gestión de archivos universitarios. Multiciencias. Vol. 14 No. 2 México (Pp. 152-161).

Paz, Luis Ernesto; del Sol, Beatriz. (2017). Conservación preventiva de documentos fotográficos con valor patrimonial. Métodos de información. Vol. 8 No. 14 España(Pp. 89-109). DOI: http://dx.doi.org/10.5557/IIMEI8-N14-089109

Paz, Luis Ernesto; Hernández, Eduardo Alejandro; Izquierdo, Marlies; Brito, Ledia. (2016). Fondos documentales del Archivo Parroquial de la Iglesia Católica San Pedro y San Pablo de Corralillo. Bibliotecas. Anales de Investigación. Vol. 12 No. 1, Cuba (Pp. 41-51).

Prado, Miguel Ángel;Esteban, Miguel Ángel. (2016). Propuesta de un modelo de Sistema Integrado de Gestión de la Información Documental para las organizaciones. Revista General de 
Información y Documentación.Vol. 26 No. 2 España (Pp. 387-415). DOI: http://dx.doi.org/10.5209/RGID.54708

Puerta, Mairelys. (2016). Diagnóstico del estado de la Gestión Documental en la Universidad de La Habana. Universidad de La Habana. Cuba.

Ramírez, José Antonio. (2015). Evolución reciente y contribución de la gestión de documentos a las políticas de transparencia y las estrategias de gobierno abierto en México. Acervo. Vol. 28 No. 2Brasil (Pp. 134-146).

Real, Silvia; Bateoja, Hilda Esther. (2018). Nuevo Sistema de Gestión Documental. Revista Hallazgos. Vol. 21 No. 3 Argentina(Pp. 1-10). DOI: http://doi.org/10.15332/2422409X

Ríos, Jan. (2016). Diagnóstico de la Gestión Documental en los Órganos de la Administración Central del Estado Cubano. Universidad de La Habana. Cuba.

Ruiz, María de los Ángeles; Bodes, Ariel. (2014). La Gestión Documental y su Impacto en el Sector Empresarial Cubano. GECONTEC: Revista Internacional de Gestión del Conocimiento y la Tecnología, Vol. 2 No. 1 España (Pp. 60-75).

Sebastián, Mercedes;Martínez, Sara. (2016). Gobierno y Parlamento abierto: la participación ciudadana en el tratamiento y visualización de la información pública. Revista Interamericana de Bibliotecología. Vol. 39 No. 1, Colombia (Pp. 47-56). DOI: http://doi.org/10.17533/udea.rib.v39n1a06

Sierra, Luis Fernando. (2012). Gestión Documental enfocada a procesos: una mirada desde la administración pública distrital. Revista Interamericana de Bibliotecología, Vol. 35 No. 3, Perú (Pp 243-255).

Sobhani, Farid Ahammad;Zainuddin, Yuserrie; Amran, Azlan; Baten, Azizul.(2011). Corporate Sustainability Disclosure Practices of Selected Banks: A Trend Analysis Approach. African Journal of Business Management.Vol. 5 No. 7 Sudáfrica(Pp. 2794-2804). DOI :http://dx.doi.org/10.5897/AJBM10.1155

Sour, Laura. (2017). Gobierno electrónico y corrupción en México (2005-2010). Innovar. Vol. 27 No. 66México (Pp. 123-136). DOI: http://dx.doi.org/10.15446/innovar.v27n66.66807

Triana, Yuri; Febles, Juan Pedro; Mena, Mayra Marta; González, Neilys; García, Máryuri. (2018). Diagnóstico de los sistemas de gestión documental para desarrollar la gestión del conocimiento. Ingeniería Industrial, Vol. 39 No. 1 Cuba (Pp. 46-55). 